STRUCTURAL BIOLOGY

ISSN 2059-7983

Received 17 April 2019

Accepted 27 December 2019

Edited by G. Kurisu, Osaka University, Japan

Keywords: noncrystallographic symmetry; NCSconstrained map; direct density optimization; twinning; REFMAC5.

Supporting information: this article has supporting information at journals.iucr.org/d

Direct density-optimization method

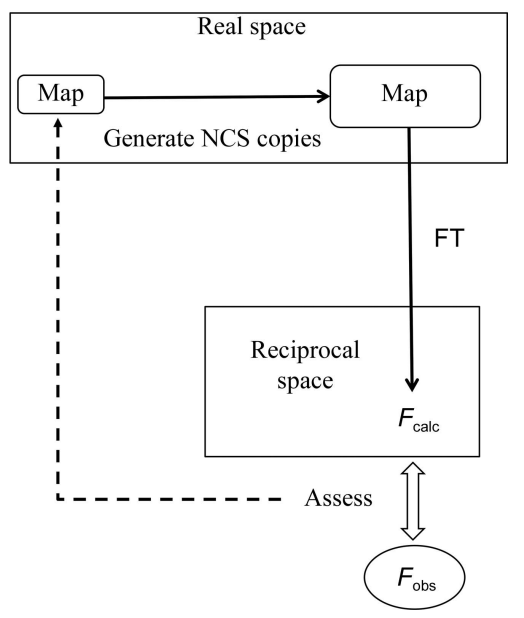

OPEN $\odot$ ACCESS

\section{Noncrystallographic symmetry-constrained map obtained by direct density optimization}

\author{
Masato Yoshimura, ${ }^{a}$ Nai-Chi Chen, ${ }^{a}$ Hong-Hsiang Guan, ${ }^{a}$ Phimonphan \\ Chuankhayan, ${ }^{a}$ Chien-Chih Lin, ${ }^{a}$ Atsushi Nakagawa ${ }^{b}$ and Chun-Jung Chen ${ }^{a, c, d *}$
}

aLife Science Group, Scientific Research Division, National Synchrotron Radiation Research Center, 101 Hsin-Ann Road, Hsinchu 30076, Taiwan, 'b Institute for Protein Research, Osaka University, 3-2 Yamadaoka, Suita, Osaka 565-0871, Japan, ' Department of Biotechnology and Bioindustry Sciences, National Cheng Kung University, Tainan 701, Taiwan, and ${ }^{\mathbf{d}}$ Department of Physics, National Tsing Hua University, Hsinchu 30043, Taiwan. *Correspondence e-mail: cjchen@nsrrc.org.tw

Noncrystallographic symmetry (NCS) averaging following molecular-replacement phasing is generally the major technique used to solve a structure with several molecules in one asymmetric unit, such as a spherical icosahedral viral particle. As an alternative method to NCS averaging, a new approach to optimize or to refine the electron density directly under NCS constraints is proposed. This method has the same effect as the conventional NCS-averaging method but does not include the process of Fourier synthesis to generate the electron density from amplitudes and the corresponding phases. It has great merit for the solution of structures with limited data that are either twinned or incomplete at low resolution. This method was applied to the case of the $T=1$ shell-domain subviral particle of Penaeus vannamei nodavirus with data affected by twinning using the REFMAC5 refinement software.

\section{Introduction}

The molecular-averaging method in real space coupled with solvent flattening is powerful in phase determination or phase improvement in protein crystallography. In the structure determination of icosahedral viruses, noncrystallographic symmetry (NCS) averaging with phase extension is a common procedure for phase improvement after initial calculations based on molecular replacement (MR) using a density map from a cryo-electron microscope, a similar structural model or initial experimental phases from isomorphous replacement or anomalous dispersion (Arnold et al., 1987). Under the special conditions that the envelope or icosahedral matrices are given with sufficient precision and the degrees of freedom of the density are sufficiently small, i.e. a lower crystallographic free fraction, the map can be built ab initio (Yoshimura et al., 2016). Since early in the 1970s, molecular averaging has been performed with iterative calculations of Fourier transformation (FT) and inverse FT between real and inverse space (Buehner et al., 1974; Bricogne, 1976). Many applications and results using iterative molecular-averaging methods have been reviewed by Kleywegt \& Read (1997).

As an alternative method, we have conceived a method to optimize or refine the density values directly under NCS constraints to reproduce the observables of the amplitude $F_{\text {obs }}$. We know empirically that such a direct density-optimization (DDO) method has the weakness of a small convergence radius and difficulty in finding the initial conditions to achieve the correct solutions, but once the initial conditions have been obtained, it has a good potential to attain the most reasonable solution. In contrast to the conventional averaging method, 


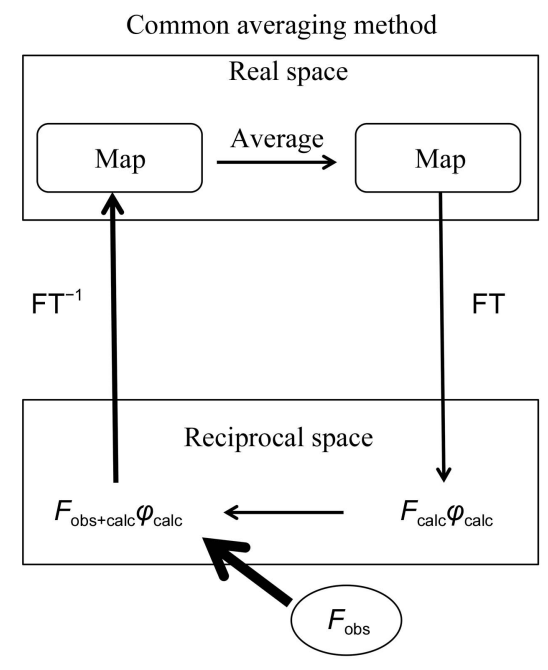

(a)
Direct density-optimization method

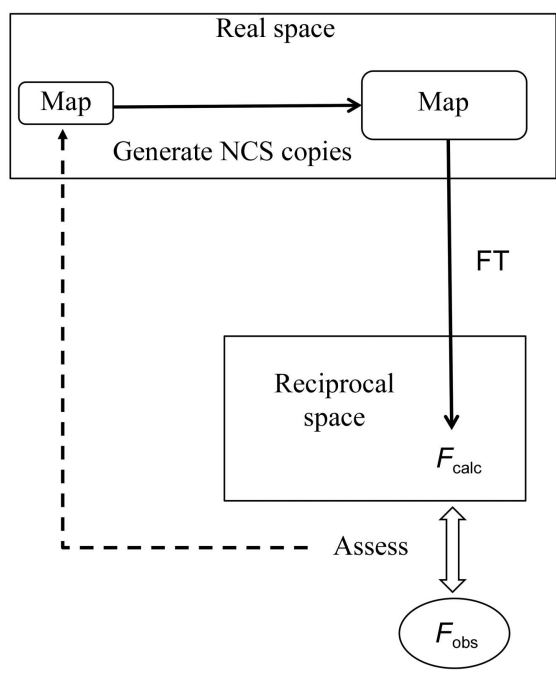

(b)

Figure 1

Schematic diagrams for the two types of calculations: $(a)$ conventional iterative methods for NCS averaging and $(b)$ the direct density-optimization (DDO) method to generate the NCS-constrained map.

Direct density optimization method for twinned case

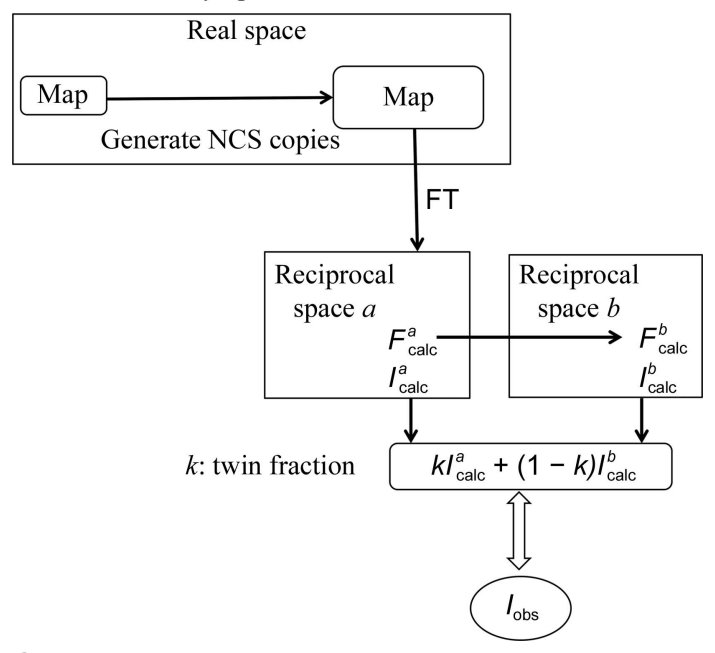

Figure 2

Schematic diagram for the direct density-optimization (DDO) method in the case of twinning.

the DDO method contains no Fourier synthesis, in which the electron density is calculated from the amplitudes (usually the observables $F_{\text {obs }}$ ) and the corresponding phases. Consequently, the electron-density maps do not suffer from incomplete observables, which are typically caused by the experimental setup, such as a cut by the beamstop shadow. Furthermore, in cases of twinned data there is significant merit in avoiding the process of Fourier synthesis, in which detwinning (or deconvoluting) the amplitudes is generally difficult.

In this paper, we report the principles and the application of the DDO method to construct an NCS-constrained density map of the $T=1$ shell-domain (S-domain; $\mathrm{Sd}$ ) subviral particle (SVP) of Penaeus vannamei nodavirus $(P v \mathrm{NV})$ in order to solve its structure. The biological details and structural results of $P v \mathrm{NV}$ have recently been reported (Chen et al., 2019). We obtained two crystal forms, in space groups $P 2_{1} 2_{1} 2_{1}$ and $P 2_{1} 3$, for the $T=1 P v \mathrm{NV}$ S-domain SVP, where the data from the $P 2_{1} 3$ crystal were merohedrally twinned. The coordinates of the $T=1 \mathrm{~S}$-domain SVP of Grouper nervous necrosis virus (GNNV; PDB entry 4rft; Chen et al., 2015) served as an initial model; the NCS-constrained electron-density maps of the two crystal forms were obtained using the DDO method. Using the NCS-constrained maps, a new structure of the $T=1 P v \mathrm{NV}$ $\mathrm{S}$-domain SVP was determined. Even though one data set was twinned, the electron-density map was deduced. In the following, we discuss the DDO method, the conditions for acquiring valid electron density and the convergence radius, and compare the DDO method with other common methods.

\section{Concept and methods}

\subsection{Concept}

Molecular averaging in real space is generally performed with an iterative calculation between real and inverse space linked by Fourier transformation (FT) and inverse FT (FT $\left.{ }^{-1}\right)$. A schematic diagram of this common method is shown in Fig. 1(a). Here, we propose a new method according to which the density of the minimal region for a target molecule is first postulated and copies of NCS-equivalent molecule densities are then generated. The calculated amplitudes $\left(F_{\text {calc }}\right)$ produced by FT are eventually compared with the observed amplitudes $\left(F_{\text {obs }}\right)$, as shown in Fig. 1(b). To maximize the agreement between the calculated $F_{\text {calc }}$ and the data $F_{\text {obs }}$, the density of the molecule is hence treated as a parameter and optimized. This method is free from the Fourier synthesis $\left(\mathrm{FT}^{-1}\right)$ process that uses the data amplitude $F_{\text {obs }}$ (Fig. 1). It has the merit of treating twinned data and solving the structure without difficulty (Fig. 2). This concept can be recognized as a 'refinement' technique that is directly applied to the electron 
density. To obtain valid electron density, the correct region of the target molecule, the correct NCS matrices and a sufficiently small crystallographic free fraction (ff; Yoshimura et al., 2016), which denotes the ratio of the unconstrained density region to the unit-cell volume, are essential. These conditions are described in Section 4. Table 1 lists notations for and definitions of the terms and abbreviations used in this work.

\subsection{Method for DDO calculations}

As there was no suitable DDO software for us to directly optimize or to refine the electron-density values in this work, we utilized the popular refinement program REFMAC5 (Murshudov et al., 2011) to perform and test the DDO method in an indirect way. REFMAC5 refines the coordinate parameters and temperature factors in seeking the maximum likelihood to reproduce the amplitude observables. The solution from the MR method was used for the initial density. The solution using the GNNV SVP retained the icosahedral relation. An icosahedral unit of the molecule was selected; the other molecules were expressed with each NCS matrix, and the NCS matrices were used as the NCS-constraint parameters for the refinement. In the case of our icosahedral virus structures, strict icosahedral matrices were constantly applied as the NCS matrices. Using REFMAC5, the coordinates and $B$ factors of the initial model were refined under unrestrained refinement, while imposing NCS constraints using the ncsconstraint option. Using unrestrained refinement that is free of any restriction by local geometry restraints, such as bond lengths and bond angles, the common case is to move atom positions that are meaninglessly dispersed because of the overfitting condition. In our case of unrestrained refinement while imposing many NCS constraints, atomic coordinates were also dispersed such that the atoms were no longer present at the actual atomic positions. Nevertheless, when the $R$ factor was decreased sufficiently even under many NCS constraints, we obtained interpretable electron density that was generated with the dispersed coordinates and $B$ factors of atoms. The obtained density map, which was generated with calculated amplitudes $F_{\text {calc }}$ and phases, was determined as an 'NCS-constrained map'. If necessary, the parameters of the NCS matrices can be refined by monitoring the decreasing $R$ factors.

\subsection{Method for purification, crystallization and data collection}

Orthorhombic $\left(P 2_{1} 2_{1} 2_{1}\right)$ and cubic $\left(P 2_{1} 3\right)$ crystals of $\Delta \mathrm{N}$ ARM $T=1 P v \mathrm{NV}$ S-domain SVP, hereafter denoted $T=1$ $P v \mathrm{NV}$-Sd, were obtained by the hanging-drop vapourdiffusion method. Drops containing the $T=1 \mathrm{~S}$-domain subparticle (30 mg ml$)^{-1}$ in $50 \mathrm{~m} M$ HEPES pH 7.4, $300 \mathrm{~m} M$ $\mathrm{NaCl}, 5 \mathrm{mM} \mathrm{CaCl}_{2}$ ) were allowed to equilibrate at $18^{\circ} \mathrm{C}$ against reservoirs consisting of (i) $0.1 M$ Tris $\mathrm{pH} 8.0,20 \%(w / v)$ poly(acrylic acid sodium salt) 5100 or (ii) $0.1 M$ Tris $\mathrm{pH} 7.8$, $0.2 M$ L-arginine, $8 \%(w / v)$ poly- $\gamma$-glutamic acid. All crystals grew after two weeks. X-ray diffraction data from these two crystal forms, cryoprotected with $20 \%$ glycerol, were collected
Table 1

Abbreviations, special terms and their definitions and explanations.

Abbreviations.

\begin{tabular}{lll}
\hline $\begin{array}{l}\text { DDO } \\
P v N V\end{array}$ & $\begin{array}{l}\text { Direct density optimization } \\
\text { Penaeus vannamei nodavirus }\end{array}$ & $\begin{array}{c}\text { New virus to be solved in this } \\
\text { work }\end{array}$ \\
GNNV & Grouper nervous necrosis virus & $\begin{array}{c}\text { Similar virus model used in MR } \\
\text { phasing }\end{array}$ \\
$\begin{array}{l}\text { SVP } \\
P v N V-S d\end{array}$ & $\begin{array}{l}\text { Subviral particle } \\
P v \text { NV shell-domain (S-domain) }\end{array}$ & $\begin{array}{c}\text { New structure to be solved in } \\
\text { this work }\end{array}$ \\
ff & Crystallographic free fraction $\dagger$ & \\
\hline
\end{tabular}

Special terms.

\begin{tabular}{ll}
\hline $\mathrm{ff}$ & Ratio of unconstrained region volume to the total unit cell $\dagger$ \\
$R$ factor & $R$ factor with $F_{\text {calc }}$ from model \\
$R_{\mathrm{d}}$ factor & $R$ factor with $F_{\text {calc }}$ from electron-density map. In this work, it \\
is calculated using a dummy-atoms model. The derivation is & the same as that of the $R$ factor.
\end{tabular}

$\dagger$ Yoshimura et al. (2016).

at 100 or $110 \mathrm{~K}$ on beamline BL44XU at SPring-8, Harima, Japan and beamline TPS 05A at NSRRC, Hsinchu, Taiwan. The data were indexed and processed using $H K L-2000$ (Otwinowski \& Minor, 1997). The data resolutions for the $P 2_{1} 2_{1} 2_{1}$ and $P 2{ }_{1} 3$ crystals was 3.38 and $3.30 \AA$, respectively, in the initial phasing stage. The data with the best resolution of $3.12 \AA$ for the $P 2_{1} 2_{1} 2_{1}$ crystal form were obtained later during the final structure-refinement stage for the structure report (Chen et al., 2019). The data statistics are shown in Table 2.

\section{Results}

\subsection{Single-crystal data in space group $P 2_{1} 2_{1} 2_{1}$}

Initial phasing of the data in space group $P 2{ }_{1} 2_{1} 2_{1}$ was performed by the MR method using the structure of $T=1$ GNNV SVP (PDB entry 4rft) as the initial model (Chen et al., 2015) in Phaser (McCoy et al., 2007). One $T=1$ SVP, which consists of 60 copies of the S-domain, was estimated to be included in the NCS unit. Phaser found an MR solution that showed a log-likelihood gain (LLG) score of 570 (the LLG before refinement was 35) on inputting an identity parameter (IDENT) of 0.5 . The initial weighted $\left(2 F_{\mathrm{o}}-F_{\mathrm{c}}\right)$ map with the MR phases was completely uninterpretable (Supplementary Fig. S1). From the initial MR solution of the SVP of GNNV, we obtained the initial 60-fold NCS matrices that maintained the strict icosahedral form. Using the REFMAC5 refinement software (Murshudov et al., 2011), the atomic coordinates and temperature factors of a single S-domain (a minimal icosahedral unit) were optimized by 'unrestrained refinement' while imposing 60-fold 'constrained' NCS matrices. The number of non-H atoms in the GNNV S-domain was 1248. After the unrestrained refinement, the atomic coordinates of the GNNV S-domain completely dispersed: almost all of the atomic coordinates did not reflect the new correct atom positions. Despite the dispersed atomic coordinates, 
Table 2

Data collection and processing.

Values in parentheses are for the outer shell.

\begin{tabular}{|c|c|c|c|}
\hline & \multicolumn{2}{|c|}{$T=1 \Delta \mathrm{N}-\mathrm{ARM} P v \mathrm{NV}-\mathrm{Sd} \mathrm{SVP}$ (PDB entry 5yl1) } & \multirow[b]{2}{*}{$T=1 P v \mathrm{NV}-\mathrm{Sd} \mathrm{SVP}$} \\
\hline & Phasing & Refinement & \\
\hline \multicolumn{4}{|l|}{ Data collection } \\
\hline Diffraction source & BL44XU, SPring-8 & TPS 05A, NSRRC & BL44XU, SPring-8 \\
\hline Wavelength $(\AA)$ & 0.9792 & 1.0000 & 0.9000 \\
\hline Temperature (K) & 100 & 110 & 100 \\
\hline Detector & MX300HE & MX300HS & MX300HE \\
\hline Space group & $P 2_{1} 2_{1} 2_{1}$ & $P 2_{1} 2_{1} 2_{1}$ & $P 2_{1} 3$ \\
\hline$a, b, c(\AA)$ & $191.54,196.86,437.17$ & $196.98,200.32,419.29$ & 244.87 \\
\hline Resolution range $(\AA ̊)$ & $42.9-3.38(3.56-3.38)$ & $30-3.12(3.23-3.12)$ & $30-3.30(3.42-3.30)$ \\
\hline No. of unique reflections & 206891 & 274292 & 69447 \\
\hline Completeness (\%) & $89.4(6.0)$ & $99.7(99.8)$ & $94.5(69.3)$ \\
\hline Multiplicity & $6.1(1.5)$ & $5.0(5.0)$ & $5.6(5.0)$ \\
\hline$\langle I / \sigma(I)\rangle$ & $6.0(1.4) \dagger$ & $7.1(1.7) \dagger$ & $4.8(1.9)$ \\
\hline \multicolumn{4}{|l|}{ Refinement } \\
\hline$R_{\text {work }} / R_{\text {free }}(\%)$ & & 21.9/22.1 & \\
\hline \multicolumn{4}{|l|}{ No. of non-H atoms } \\
\hline Protein & & 1454 & \\
\hline Ions & & $2\left[\mathrm{Ca}^{2+}\right]$ & \\
\hline \multicolumn{4}{|l|}{ R.m.s. deviations } \\
\hline Bonds $(\AA)$ & & 0.011 & \\
\hline Angles $\left({ }^{\circ}\right)$ & & 1.696 & \\
\hline \multicolumn{4}{|l|}{ Average $B$ factors $\left(\AA^{2}\right)$} \\
\hline Protein & & 64.6 & \\
\hline Ion & & 73.4 & \\
\hline
\end{tabular}

$\dagger$ In the refinement, we forced 60 -fold strict icosahedral constraints. This has the same effect as averaging.

200. On monitoring the $R_{\mathrm{d}}$ factors, initial parameters, such as NCS matrices, were refined with perturbation trials and as few as ten refinement cycles. In Fig. 3, these $R_{\mathrm{d}}$ factors with initial NCS matrices and with refined NCS matrices are shown against the cycle number of the REFMAC5 refinement. When refinement of the NCS matrices was performed, the 60-fold NCS matrices were maintained to retain a strict icosahedral relation to each other; i.e. only six parameters for rotations and positions of the centre of the icosahedron to generate NCS matrices were refined. After the NCS matrices had been refined, we obtained an NCSconstrained map with an $R_{\mathrm{d}}$ factor of $23 \%$, which was generated from 'calculated amplitudes $F_{\text {calc }}$ ' and 'calculated phases'. Using the data with the highest resolution of $3.12 \AA$, the NCS matrix of the icosahedron was refined again and the final $R_{\mathrm{d}}$ factor reached $21 \%$. As the refined atomic coordinates did not reflect the actual atomic positions of the

REFMAC5 showed a reasonable $R$ factor; in this case it was less than $25 \%$. The $R$ factor typically shows the agreement of the calculated amplitudes from the atomic model with the diffraction data. In this work, we name the $R$ factor the ' $R_{\mathrm{d}}$ factor' to represent the agreement of the calculated amplitudes from the electron density, instead of the atomic model, with the observed data.

The number of unrestrained refinement cycles was extended as long as the $R_{\mathrm{d}}$ factor decreased and converged to a minimum value. The number of cycles was usually less than

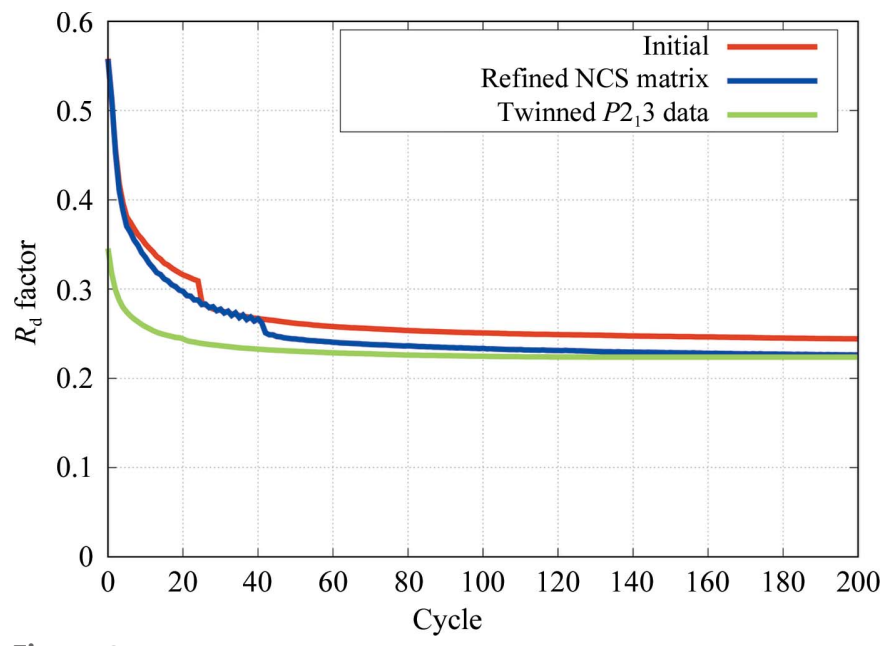

Figure 3

$R_{\mathrm{d}}$ factors plotted against refinement cycles. The red line shows the results with the initial NCS matrices for the single-crystal $P 2_{1} 2_{1} 2_{1}$ data. The blue line shows the results with the refined icosahedral NCS matrices. The green line shows the results with the twinned $P 2{ }_{1} 3$ data. All results are for data at the phasing stage. structure, we call these refined atoms 'dummy atoms'. The dispersed dummy atoms that served the original MR model are shown in Fig. 4(a) with the obtained NCS-constrained map. With the NCS-constrained map, the new structure of the $T=1 P v \mathrm{NV}-\mathrm{Sd}$ particle in the icosahedral unit could be manually built with Coot (Emsley et al., 2010). The built model in the icosahedral unit underwent restrained refinement under the final NCS constraints that maintained strict icosahedral relations (PDB entry 5yl1). The $R_{\text {work }}$ and $R_{\text {free }}$ of the final model were $21.9 \%$ and $22.1 \%$, respectively (Chen et al., 2019; Table 2). The main chains of the initial MR model $(T=1$ GNNV SVP; PDB entry 4rft), the final refined structure model $(T=1 P v \mathrm{NV}-\mathrm{Sd})$ and the NCS-constrained map are shown in Fig. 4(b).

\subsection{Twinned crystal data in space group $P 2_{1} 3$}

According to the statistics of the amplitudes and the icosahedral-related peaks from the self-rotation function, the data in space group $P 2{ }_{1} 3$ were expected to be merohedrally twinned. All estimators of twinning obtained using TRUN$C A T E$ in the CCP4 software package (Winn et al., 2011) showed that the data were completely twinned; for example, an $L$ statistic of 0.339 (Padilla \& Yeates, 2003). The selfrotation function showed two directions of icosahedral symmetry, with each orientation related by a $90^{\circ}$ rotation. From the crystal symmetry and packing analysis, we located the centres of four presumed particles at $(1 / 4,1 / 4,1 / 4)$, $(3 / 4,3 / 4,1 / 4),(1 / 4,3 / 4,3 / 4)$ and $(3 / 4,1 / 4,3 / 4)$. One-third of the $T=1 \mathrm{SVP}$, which contains 20 copies of $P v \mathrm{NV}-\mathrm{Sd}$, was present in the NCS unit. 
Using the refined dummy atoms, which contained the information from the electron density of $P v \mathrm{NV}-\mathrm{Sd}$ but in

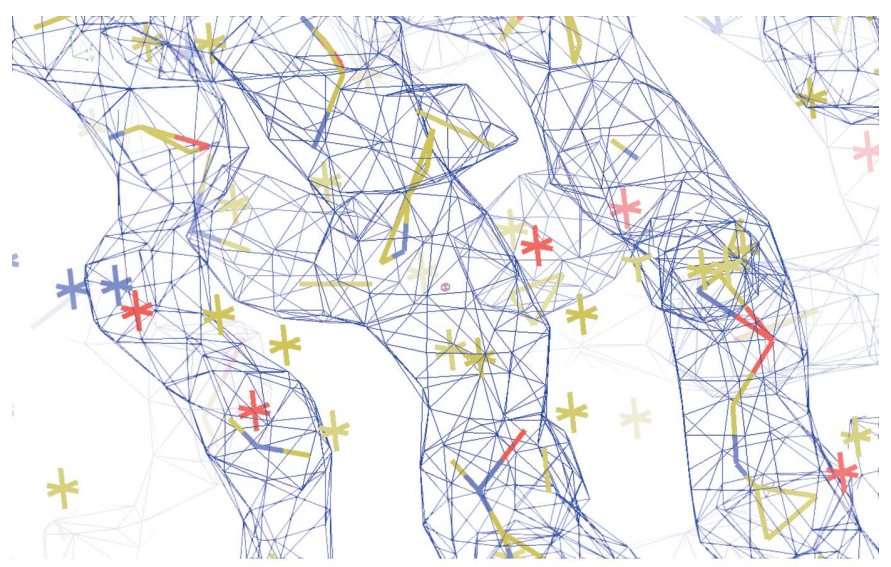

(a)

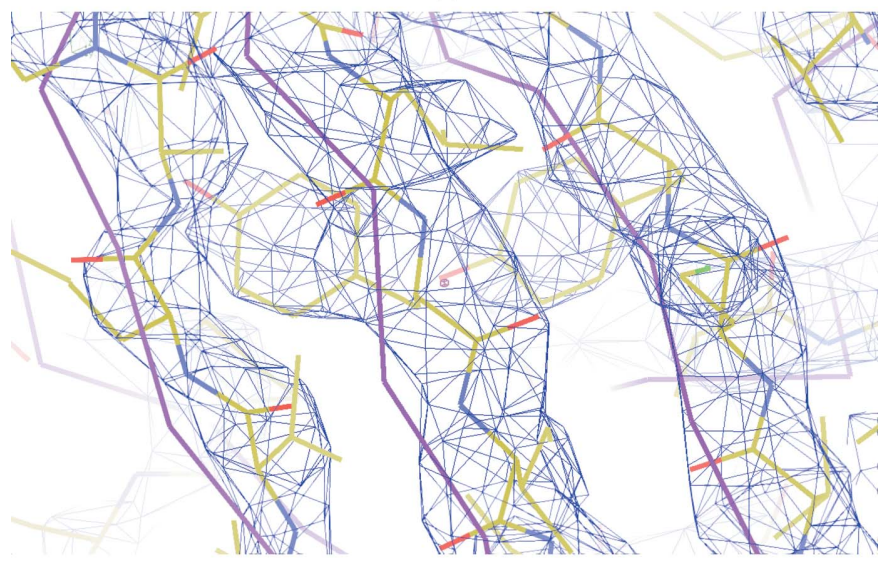

(b)

Figure 4

(a) The NCS-constrained electron-density map with dummy atoms that are fully dispersed from the actual atomic positions (yellow sticks) contains the correct electron-density information. (b) The NCSconstrained map with the main-chain trace of the initial model of GNNV (purple) and the final structural model of $P v \mathrm{NV}-\mathrm{Sd}$ (yellow sticks) fitted to the map as a guide for the eye. Using the NCS-constrained map, the structure model was built. The bias from the initial MR model can be removed. The contour level of the map is $2.3 \sigma$.

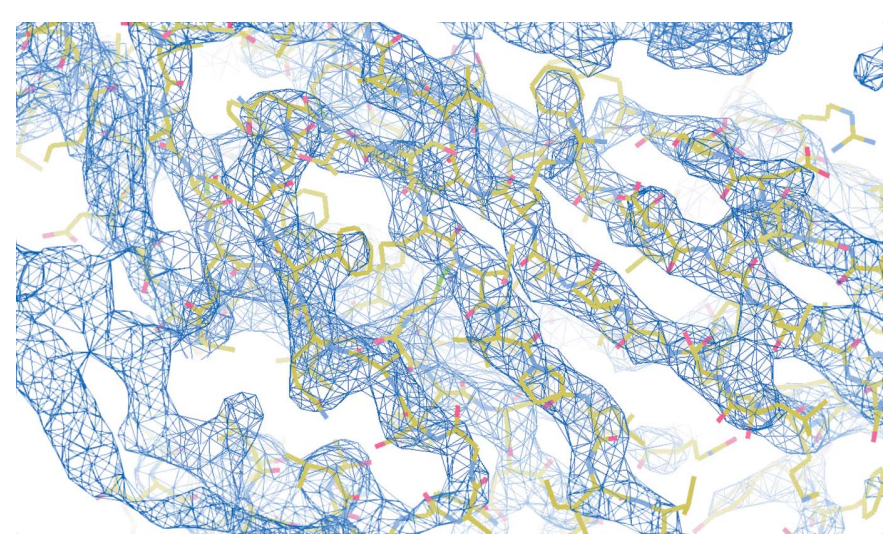

Figure 5

The NCS-constrained map from the merohedrally twinned data. The map is interpretable and clear despite the twinning. The contour level of the map is $2.0 \sigma$. The final refined structure model of $P v \mathrm{NV}-\mathrm{Sd}$ fitted to the map is shown in yellow as a guide for the eye. which the atomic positions seemed to be dispersed, the initial phases from MR for the twinned data from the $P 2_{1} 3$ crystal were obtained with a high LLG score (IDENT $=0.8$ ) that was as large as 10000 on refining the rotations and translations of 20 initial molecules with refinement and phasing by Phaser. As a trial, using the $T=1$ GNNV SVP as the initial structural model, Phaser picked very low LLG scores of $\sim 20$ (IDENT $=$ 0.8 ). One dummy-atom molecule of $P v \mathrm{NV}-\mathrm{Sd}$ from the MR solution was selected; its NCS matrix was determined as a unit matrix. Using the other 19 NCS-related molecules of the solution, the 19 NCS matrices were calculated. The DDO method only has a convolution process of amplitude calculation for twinned observables. REFMAC5 has a refinement function for the twinning fraction (see Section 2.3 of Murshudov et al., 2011); this showed that the twinning operator was $(k, h,-l)$ against $(h, k, l)$. Using REFMAC5, the dummy atoms were subjected to unrestrained refinement with 20 NCS constraints and with twinning-fraction refinement. The $R_{\mathrm{d}}$ factor of the unrestrained refinement reached $22.4 \%$ (Fig. 3). The twinning fractions were 0.69 and 0.31 in the final refinement cycle. The NCS-constrained maps for twinned data with the final refined model of $P v \mathrm{NV}-\mathrm{Sd}$ are shown in Fig. 5.

\section{Discussion}

\subsection{Direct density optimization and REFMAC5}

Since the early years, NCS has been known to be powerful in phase improvement (Main \& Rossmann, 1966; Bricogne, 1976; Arnold \& Rossmann, 1986; Rossmann, 1990), and has assisted in many cases of phase improvement using several programs, such as $D M$ (Cowtan \& Main, 1996) in the CCP4 suite and RESOLVE (Terwilliger, 2002) in Phenix (Liebschner et al., 2019). Recent attention has been paid to real-space refinement because of the requirements of cryo-EM structure refinement (Afonine et al., 2018). In almost all cases, the imposition of NCS has been performed more or less using the iterative or averaged method shown in Fig. 1(a). Fourier synthesis calculations (or inverse Fourier transformations; $\mathrm{FT}^{-1}$ ) are required to process the map, whereas the DDO method has no Fourier synthesis calculation (Fig. 1b). A map generated using Fourier synthesis calculations could be affected by defects in the data. The strength of the DDO method is its robustness against defects in the data $F_{\mathrm{obs}}$, such as statistical errors, systematic noise and incompleteness. Especially in cases of twinning, the decomposition of amplitudes is a difficult task in the Fourier synthesis calculations in the common iterative-averaging method. The DDO method has a great strength in avoiding this difficulty in the decomposition of observables.

To execute the DDO method, we used the refinement function of REFMAC5, which refines the atomic coordinates and their temperature factors. Our initial intent was to seek to undertake refinement of the temperature factors or occupancies of fixed coordinate atoms, which served to refine densities on the map grid positions. We found, unexpectedly, that flexible atomic coordination was not a problem in 
obtaining correct densities under the conditions described in the next section. We previously reported that the envelope of the region is important for NCS averaging (Yoshimura et al., 2016). The condition that we can refine the coordinates of density grid points means that we can refine the envelope itself with increased flexibility.

\subsection{Conditions for obtaining a correct map}

In our previous work (Yoshimura et al., 2016), we introduced the crystallographic free fraction (ff), which gives the ratio of the unconstrained density region to the unit cell. When $\mathrm{ff}$ is sufficiently small, which is the condition of overdetermination, the density of the unconstrained region is determined uniquely. We showed an empirical ff value of $<0.1$ to be the criterion for a feasible $a b$ initio condition using the iteration-averaging method. In this work, we show the same situation for the feasible direct determination of the density. The values of $\mathrm{ff}$ are $<1 / 60$ for the $P 2_{1} 2_{1} 2_{1}$ data and $<1 / 20$ for the twinned $P 2_{1} 3$ data; the inequality arises from considering the constraint on the solvent region. Both ff values are sufficiently small. Even the number of dummy atoms (1248), which was the original number of non- $\mathrm{H}$ atoms in the GNNV S-domains, has an additional degree of freedom of the $x, y$ and $z$ coordinates in the REFMAC5 refinement. The condition of over-determination remained satisfied when the number of variables $(4996 ; 1248 \times 4)$ was $1 / 21$ times the number of unique observables (206 891) in the $P 2_{1} 2_{1} 2_{1}$ data. Two further conditions, the correct NCS matrices and NCS minimal regions and the acquisition of a global minimum unique solution, are also required to derive a correct map. We summarize these three points as

(i) correct NCS matrix and region,

(ii) sufficiently small ff,

(iii) acquisition of a global minimum solution.

When the value of $\mathrm{ff}$ is not sufficiently small but satisfies $\mathrm{ff}<$ 0.5 , even on obtaining a global minimum solution or a unique solution (Millane \& Arnal, 2015) care should be taken in obtaining the density map in order to avoid it being ill-posed through errors in the data.

To examine whether a global minimum solution can be obtained, we refer to the metric of agreement between the calculated amplitudes and observables as the $R_{\mathrm{d}}$ factor; we can then evaluate the solution by inspecting whether or not the electron-density map is interpretable. A starting point or an initial condition from which the global minimum can be attained is referred to as 'in the convergence radius'. When the parameter optimization falls into a local minimum or a shortage of the degree of freedom of the parameters occurs, the third condition of the acquisition of a global minimum solution is not satisfied.

\subsection{Convergence radius of the DDO method}

In contrast to the iteration-averaging methods, the convergence radius of direct density refinement is empirically expected to be small. The small values of $\mathrm{ff}$ and the precise icosahedral NCS matrices guided the derivation of the correct density. Fortunately, we attained a global minimum solution for the $P 2_{1} 2_{1} 2_{1}$ single-crystal data beginning from the initial condition of the GNNV S-domain particles. So far, for the twinned $P 2_{1} 3$ data we only obtained a correct solution when using $P v \mathrm{NV}$ dummy atoms as the starting point, for which the initial $R_{\mathrm{d}}$ factor was $35 \%$ (Fig. 3). Our few trials using GNNV S-domain particles as a starting point did not succeed for the twinned data, which might reflect a small convergence radius for the DDO method. The other reason is that the twinningfraction parameter led to a moderate increase in $\mathrm{ff}(<1 / 20)$ because of the additional degrees of freedom of the variables. Phasing of the twinned crystal is a major problem to be overcome (Ginn \& Stuart, 2016; Sabin \& Plevka, 2016); we need to further investigate how to increase the convergence radius to obtain a global minimum solution.

\subsection{Comparison with the common iterative-averaging method}

In Fig. 6, we compare the conventional NCS-averaging map from the $D M$ software with the map from the DDO method. There is no critical difference in quality between the two maps. However, in Fig. 6(a) there are missing densities for the DM

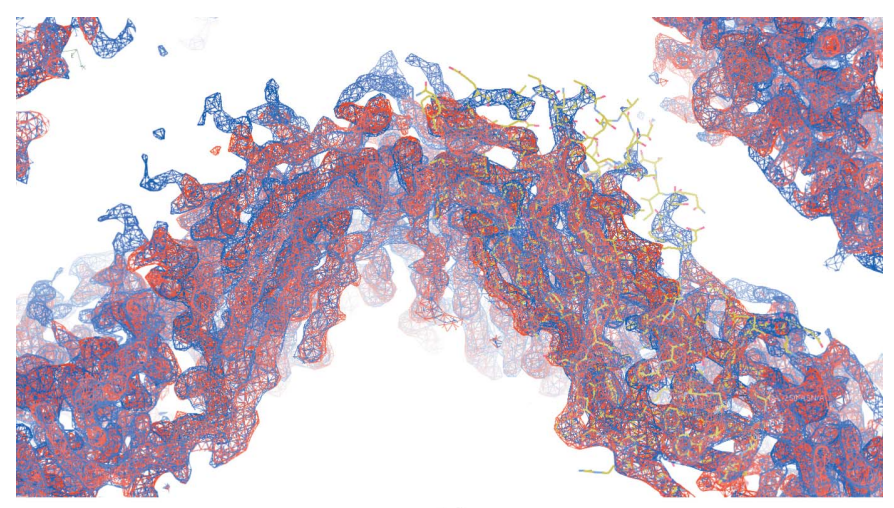

(a)

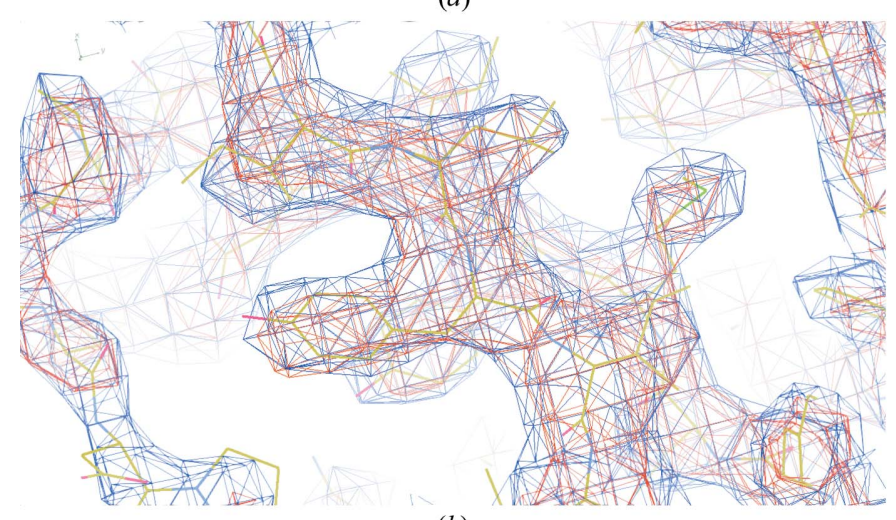

(b)

Figure 6

A comparison of the NCS-constrained map (blue mesh) with the $D M$ map (red mesh) at the same contour level $(1.5 \sigma)$. Both maps were calculated using the 3.37 A resolution data at the phasing stage and initial NCS matrices which were obtained from the MR solution. (a) shows a view of the fivefold axis of the viral particles. The outside of the particle in the $D M$ map (red) disappears owing to the initial NCS masks. (b) Both maps have the same quality; the NCS-constrained map is a little denser. The yellow stick model is the final model fitted to the map as a guide for the eye. 
Table 3

Comparisons with common methods in terms of mean phase errors and $R$ factors.

The data at the phasing stage were used in these comparisons. In the mean phase-error calculations, the refined final structure model (PDB entry 5yli) with the data at the phasing stage was used as the best phases. The structure model 5yli was refined with the data to an $R$ factor of $0.283\left(R_{\text {free }}=0.261\right)$. All $R$ and $R_{\mathrm{d}}$ factor values are taken from the results of REFMAC5. Mean phase errors were calculated using CPHASEMATCH in CCP4 (Winn et al., 2011).

\begin{tabular}{lll}
\hline Methods & $\begin{array}{l}\text { Mean } \\
\text { phase } \\
\text { error }\left(^{\circ}\right)\end{array}$ & $\begin{array}{l}R \text { or } R_{\mathrm{d}} \text { factor } \\
\left(\text { free } R \text { or } R_{\mathrm{d}} \text { factor }\right)\end{array}$ \\
\hline Molecular replacement & 76.0 & $0.559(0.561)$ \\
NCS-constrained map by DDO & 30.6 & $0.244(0.246)$ \\
$R E F M A C 5$ restrained & 52.6 & $0.465(0.470)$ \\
$D M$ & 34.1 & $-(-)$ \\
\hline
\end{tabular}

map on the outside surface of the $T=1 P v \mathrm{NV}$-Sd particle which might not be covered by the NCS mask. For $D M$, it is difficult to generate the NCS (solvent or averaging) mask at the boundary regions between neighbouring particles. The DDO method has the same function of solvent flattening as $D M$ in placing no dummy atoms in the solvent region. Such a vacant space for dummy atoms is fixed to a density value of zero. The DDO method has flexibility in the boundary-region determination because of the atom mobilities. $D M$ includes an additional function of histogram matching, but the DDO method does not apply such a process. Furthermore, $D M$ can execute averaging in a phase-extension manner to guide towards a global minimum solution, whereas no such equivalent function can be applied in the DDO method. The incorporation of phase extension into the DDO method will be our next work, in which the convergence radius will be expected to increase.

\subsection{Comparison with REFMAC5 restrained refinement}

In Fig. 7, we compare the DDO map with the resulting map from REFMAC5 refinement with the common use of coordinates restrained by the 60 -fold NCS constraint. The restrained refinement was performed with sufficient cycles (200). Although there was a structural difference of $2.7 \AA$ on average between the main chains of the initial MR model and the final built model, most fractions of the main chains of the MR model were successfully shifted and adjusted to the new correct positions after refinement. The $R$ factor had a smallest value of 0.465 when the ridge distance $\sigma$ was 0.02 (from the range 0.01-0.30) for the 'jelly-body' restraint function (see Section 2.6.5 of Murshudov et al., 2011). Most of the main chain seemed to be traceable with the weighted $\left(2 F_{\mathrm{o}}-F_{\mathrm{c}}\right)$ map by REFMAC5. Most parts of the main chains were successfully shifted to new main-chain positions, as shown in Fig. 7(a), but some parts failed to be shifted, as shown in Fig. 7(b). The NCS-constrained map from DDO can be seen to easily provide a completely new phase, removing the bias from the initial model phases by completely loosening the restraints on coordinates. In Table 3, the mean phase error and $R_{\mathrm{d}}$ factor

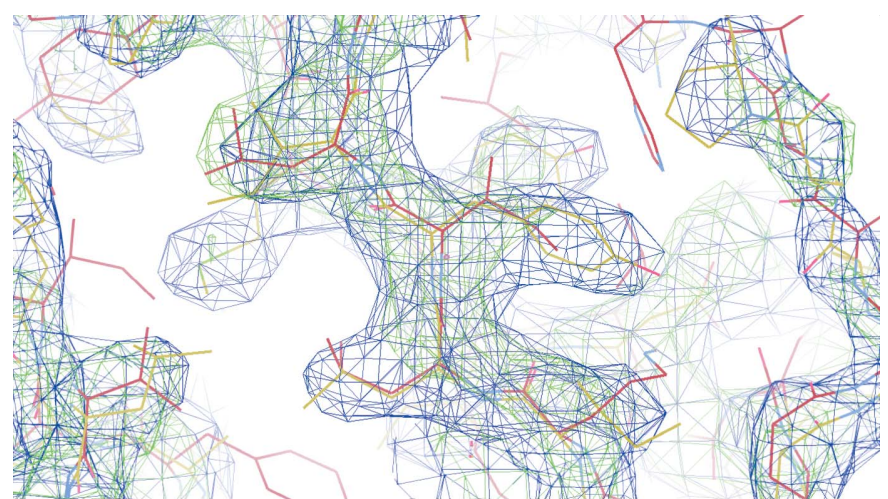

(a)

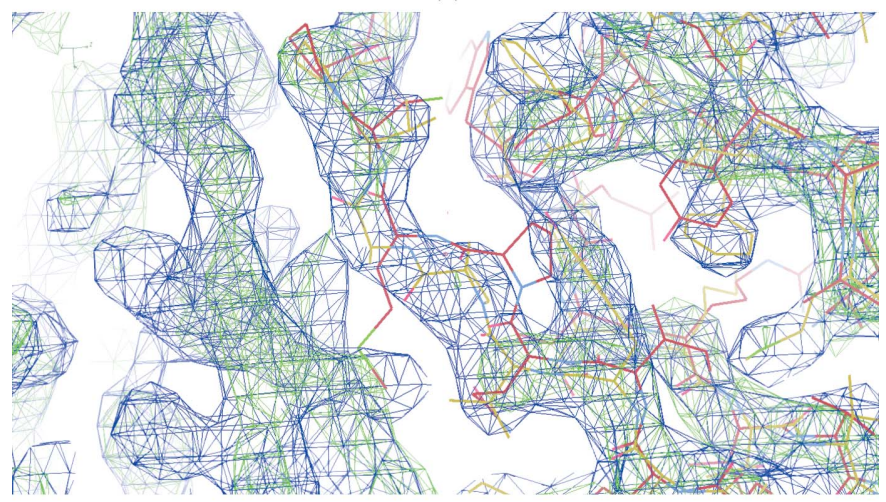

Figure 7

(b)

Comparisons of the DDO map (blue mesh) with the map resulting from REFMAC5 restrained refinement (green mesh). The REFMAC5 map (green mesh) is the weighted $\left(2 F_{\mathrm{o}}-F_{\mathrm{c}}\right)$ map. The contour levels of both maps are $2.0 \sigma$. Both maps were calculated using the $3.37 \AA$ resolution data at the phasing stage and the initial NCS matrices which were obtained from the MR solution. Yellow carbon bonds are the final model fitted to the NCS-constrained map. Brown carbon bonds are the result of REFMAC5 restrained refinement. $\mathrm{C}^{\alpha}$ displacements between the final fitted model and the MR solution are $2.7 \AA$ on average. Most of the refined main chains (brown) jumped to fit to the new position, as shown in (a). However, some parts of the refined main chains failed to fit the new map, as shown at the centre of $(b)$. Even though both maps were calculated using the same initial structure, the NCS-constrained map from DDO is free from bias from the initial structure. The yellow stick model is the final model fitted to the map as a guide for the eye.

for each method are summarized, showing that the removal of bias in the DDO method and $D M$ is of comparable quality.

\section{Conclusions}

The use of NCS is powerful for phasing or phase improvement. NCS has been used as an averaging method in real space. We propose an NCS-constrained map with a new method of direct density optimization (DDO). Whereas the NCS-constrained map is equivalent to an NCS-averaging map, the DDO method requires no Fourier synthesis that calculates a map from $F_{\text {obs }}$ and its phases. To perform DDO, we used unrestrained refinement in REFMAC5 while imposing NCS constraints, and applied this method to a new structure of $T=1$ $P v \mathrm{NV}$-Sd. With the condition that the crystallographic ff is sufficiently small, an interpretable map was obtained and the structure of $T=1 P v \mathrm{NV}-\mathrm{Sd}$ was subsequently solved. We 
further demonstrate the application of the DDO method to other $T=1 P v \mathrm{NV}-\mathrm{Sd}$ data sets that were twinned, in which the DDO method has no difficulty in generating the NCSconstrained map. A comparison of the NCS-constrained map with DDO and the map from $D M$, which uses the averaging method, shows no critical difference except that the DDO map produces more complete density at the molecular boundary. Compared with the result of REFMAC5 restrained refinement, the NCS-constrained map with DDO can easily remove the bias from the initial model with the same effort. By making the convergence radius large in future developments, the method will be more powerful for the solution of structures with a large number of NCS including twinned data.

\section{Acknowledgements}

We are indebted to the staff of beamlines TPS 05A, TLS 13B1 and $15 \mathrm{~A} 1$ at the National Synchrotron Radiation Research Center (NSRRC) in Taiwan, Eiki Yamashita at beamline BL44XU and the Taiwan beamline BL12B2 at SPring-8 in Japan for technical assistance under proposal Nos. 2015A6600, 2015A4000, 2015B6600, 2015B4004, 2015B4010, 2016A6600, 2016A6659, 201A4012, 2016B4000, 2017A4000, 2017A6600, 2017B6639, 2018A6600, 2018A6864, 2018B6600 and 2018B6864, partly supported by the International Collaborative Research Program of the Institute for Protein Research, Osaka University (ICR-16-05, ICR-17-05, ICR18-05, ICR1905). We thank Tomitake Tsukihara, Masaki Yamamoto of RIKEN and Takashi Kumasaka of JASRI and their staff for useful discussions.

\section{Funding information}

This work was supported in part by National Science Council (NSC) and Ministry of Science and Technology (MOST) grants 101-2628-B-213-001-MY4, 102-2627-M-213-001-MY3, 105-2311-B-213-001-MY3, 107-2923-B-213-001-MY3 and 1082311-B-213-001-MY3 and NSRRC grants to C-JC.

\section{References}

Afonine, P. V., Poon, B. K., Read, R. J., Sobolev, O. V., Terwilliger, T. C., Urzhumtsev, A. \& Adams, P. D. (2018). Acta Cryst. D74, 531544.
Arnold, E. \& Rossmann, M. G. (1986). Proc. Natl Acad. Sci. USA, 83, 5489-5493.

Arnold, E., Vriend, G., Luo, M., Griffith, J. P., Kamer, G., Erickson, J. W., Johnson, J. E. \& Rossmann, M. G. (1987). Acta Cryst. A43, 346-361.

Bricogne, G. (1976). Acta Cryst. A32, 832-847.

Buehner, M., Ford, G. C., Moras, D., Olsen, K. W. \& Rossmann, M. G. (1974). J. Mol. Biol. 90, 25-49.

Chen, N.-C., Yoshimura, M., Guan, H.-H., Wang, T.-Y., Misumi, Y., Lin, C.-C., Chuankhayan, P., Nakagawa, A., Chan, S.-I., Tsukihara, T., Chen, T.-Y. \& Chen, C.-J. (2015). PLoS Pathog. 11, e1005203.

Chen, N.-C., Yoshimura, M., Miyazaki, N., Guan, H.-H., Chuankhayan, P., Lin, C.-C., Chen, S.-K., Lin, P.-J., Huang, Y.-C., Iwasaki, K., Nakagawa, A., Chan, S.-I. \& Chen, C.-J. (2019). Commun. Biol. 2, 72 .

Cowtan, K. D. \& Main, P. (1996). Acta Cryst. D52, 43-48.

Emsley, P., Lohkamp, B., Scott, W. G. \& Cowtan, K. (2010). Acta Cryst. D66, 486-501.

Ginn, H. M. \& Stuart, D. I. (2016). Acta Cryst. D72, 817-822.

Kleywegt, G. J. \& Read, R. J. (1997). Structure, 5, 15571569.

Liebschner, D., Afonine, P. V., Baker, M. L., Bunkóczi, G., Chen, V. B., Croll, T. I., Hintze, B., Hung, L.-W., Jain, S., McCoy, A. J., Moriarty, N. W., Oeffner, R. D., Poon, B. K., Prisant, M. G., Read, R. J., Richardson, J. S., Richardson, D. C., Sammito, M. D., Sobolev, O. V., Stockwell, D. H., Terwilliger, T. C., Urzhumtsev, A. G., Videau, L. L., Williams, C. J. \& Adams, P. D. (2019). Acta Cryst. D75, 861-877.

Main, P. \& Rossmann, M. G. (1966). Acta Cryst. 21, 67-72.

McCoy, A. J., Grosse-Kunstleve, R. W., Adams, P. D., Winn, M. D., Storoni, L. C. \& Read, R. J. (2007). J. Appl. Cryst. 40, 658-674.

Millane, R. P. \& Arnal, R. D. (2015). Acta Cryst. A71, 592-598.

Murshudov, G. N., Skubák, P., Lebedev, A. A., Pannu, N. S., Steiner, R. A., Nicholls, R. A., Winn, M. D., Long, F. \& Vagin, A. A. (2011). Acta Cryst. D67, 355-367.

Otwinowski, Z. \& Minor, W. (1997). Methods Enzymol. 276, $307-$ 326.

Padilla, J. E. \& Yeates, T. O. (2003). Acta Cryst. D59, 1124-1130.

Rossmann, M. G. (1990). Acta Cryst. A46, 73-82.

Sabin, C. \& Plevka, P. (2016). Acta Cryst. F72, 188-197.

Terwilliger, T. C. (2002). Acta Cryst. D58, 2082-2086.

Winn, M. D., Ballard, C. C., Cowtan, K. D., Dodson, E. J., Emsley, P., Evans, P. R., Keegan, R. M., Krissinel, E. B., Leslie, A. G. W., McCoy, A., McNicholas, S. J., Murshudov, G. N., Pannu, N. S., Potterton, E. A., Powell, H. R., Read, R. J., Vagin, A. \& Wilson, K. S. (2011). Acta Cryst. D67, 235-242.

Yoshimura, M., Chen, N.-C., Guan, H.-H., Chuankhayan, P., Lin, C.-C., Nakagawa, A. \& Chen, C.-J. (2016). Acta Cryst. D72, 830840. 